\title{
A Rare Case of Pneumomediastinum after Eustachian Tube Dilation
}

\author{
Sallie Long Adetokunbo Obayemi Anaïs Rameau \\ Department of Otolaryngology, Head and Neck Surgery, NewYork-Presbyterian Hospital/Weill Cornell \\ Medical Center, New York, NY, USA
}

\section{Keywords}

Eustachian tube dilation - Balloon dilation - Eustachian tube dysfunction P Pneumomediastinum

\begin{abstract}
Eustachian tube dilation (ETD) is a relatively new intervention for the treatment of eustachian tube dysfunction. Though it previously had no assigned billing code, the American Medical Association recently accepted a new Category I Current Procedural Terminology code application for ETD to be effective in January 2021. Reported complications are uncommon and usually minor. Herein, we present a rare case of massive pneumomediastinum following this procedure. Such major complications are critical to report as ETD becomes a more commonly practiced procedure.

\section{(c) 2021 The Author(s)}

Published by S. Karger AG, Basel
\end{abstract}

\section{Introduction}

Eustachian tube dilation (ETD) can occur from either mechanical or functional abnormalities of the eustachian tube, which subsequently disrupt the pressure equalization system between the nasopharynx and the middle ear. It is a problem frequently encountered by otolaryngologists and can present with symptoms such as aural fullness, otalgia, or tinnitus [1]. Treatment options for symp-

\section{karger@karger.com www.karger.com/orl \\ Karger}

(C) 2021 The Author(s)

Published by S. Karger AG, Basel

BOPEN ACCESS

This is an Open Access article licensed under the Creative Common Attribution-NonCommercial-4.0 International License (CC BY-NC) (http://www.karger.com/Services/OpenAccessLicense), applicable to the online version of the article only. Usage and distribution for commercial purposes requires written permission. tomatic patients who fail medical management include adenoidectomy, tympanostomy tube placement, laser tuboplasty, and eustachian tube balloon dilation. ETD for the treatment of ETD is a relatively new intervention. It is performed by inserting a balloon catheter endonasally into the orifice of the eustachian tube until the bony-cartilaginous junction is reached. The balloon is then inflated inside the cartilaginous portion of the eustachian tube for up to 2 min before removal $[2,3]$.

Though it previously had no assigned billing code, the American Medical Association recently accepted a new Category I Current Procedural Terminology code application for ETD to be effective in January 2021 [4]. With this upcoming change, we expect that eustachian tube balloon dilation will be performed more widely. Although complications are uncommon and typically mild, the procedure is not always a benign intervention. Herein, we present a case of massive pneumomediastinum following this procedure for a patient with ETD. Such severe complications are critical to report as ETD becomes more commonly practiced.

\section{Case Report}

A healthy 51-year-old female with a history of ETD presented to our institution on the first postoperative day following balloon sinuplasty and bilateral ETD at an outside surgical facility. The Entellus Xpress ${ }^{\mathrm{TM}}$ ENT dilation system was insufflated to $12 \mathrm{~atm}$ with 


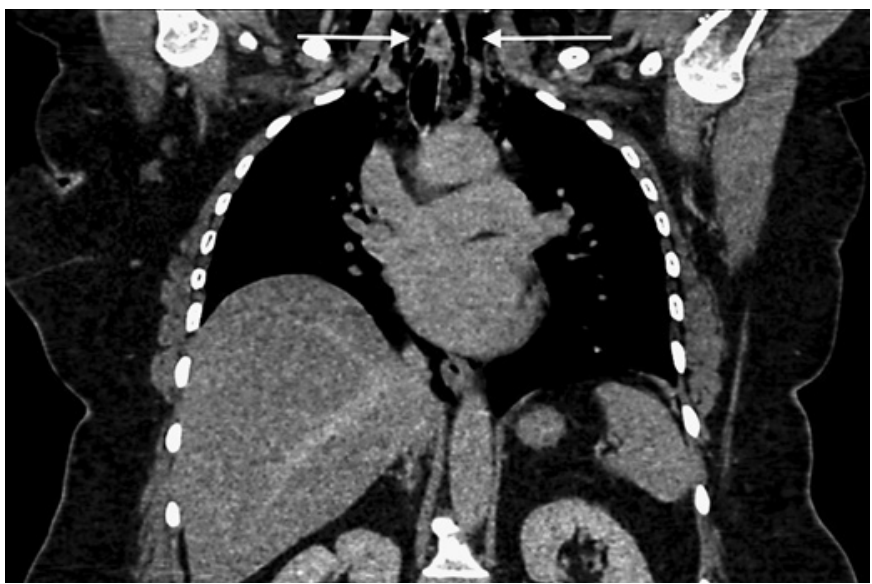

Fig. 1. Coronal CT scan of the chest showing pneumomediastinum. White arrows point to areas of air in the deep neck spaces extending inferiorly into the superior mediastinum.

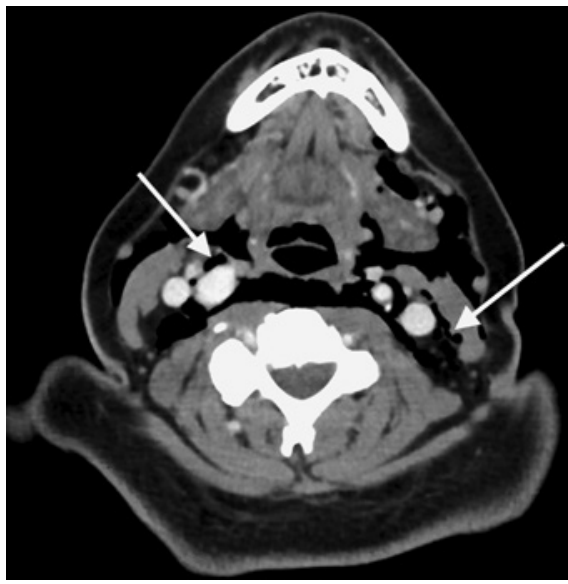

Fig. 2. Axial CT scan of the neck showing extensive soft tissue emphysema. a $6 \times 16 \mathrm{~mm}$ balloon per standard protocol, with no deviation from the manufacturer's recommended use. The procedure was uncomplicated technically, per primary surgeon's report, and immediately postoperatively the patient endorsed mild bilateral epistaxis but no discomfort. On postoperative day 1 , she reported sudden onset neck swelling, dysphagia, and pain after an episode of sneezing.

Physical examination was notable for an erythematous neck, facial swelling, trismus, and crepitus. Flexible fiberoptic laryngoscopy showed mild bloody secretions but no frank mucosal injury. A CT scan of the neck and chest showed extensive soft tissue emphysema centered at the left torus tubarius, extending into the ipsilateral retropharyngeal and carotid spaces and into the mediastinum (Fig. 1, 2).

The patient was managed conservatively with antibiotic prophylaxis and observation by the otolaryngology and cardiothoracic surgery teams. Daily chest X-rays demonstrated continued improvement of the pneumomediastinum and subcutaneous emphysema. The patient's symptoms ameliorated and she was discharged home after 3 days on oral antibiotics with no further complications.

\section{Discussion}

This case illustrates a serious complication from a seemingly minimally invasive procedure. The existing literature on eustachian tube balloon dilation suggests that the procedure is safe and effective with only a small proportion of patients experiencing any adverse effects. In a systematic review of over 1,800 procedures in 1,155 patients undergoing eustachian tube balloon dilation, Huisman et al. [1] reported improvement in both subjective and objective measures of ETD. The aggregated minor complication rate was $2 \%$ and the most common adverse event was self-limited local bleeding. Three patients had preauricular emphysema that self-resolved.

In their review of 2,272 patients undergoing balloon dilation in Germany, Skevas et al.[3] reported 10 cases of subcutaneous emphysema and 3 cases of pneumomediastinum. Many of these events occurred after maneuvers such as sneezing or nose blowing, and all occurred no later than the third postoperative day [2]. Within the US literature, Shah et al. [5] reported a case of a 28 -year-old male who presented with pneumomediastinum on the first postoperative day while engaging in heavy lifting.

These cases are similar in that they were usually preceded by an increase in endonasal pressure within the first few postoperative days. Our patient similarly presented following an episode of sneezing on the first postoperative day. Per the operating surgeon, the original procedure was uncomplicated, although the operative report was not provided. These cases highlight the critical importance of risk and benefit discussion prior to proceeding with any surgical intervention, no matter how small the perceived risks may be. Furthermore, patients should be counseled to sneeze with the mouth open, avoid heavy lifting or straining, and consider use of stool softeners to prevent constipation during the initial postoperative period.

\section{Conclusions}

Pneumomediastinum is a rare but serious complication following eustachian tube balloon dilation, with just 1 prior reported case in the USA. While this minimally 
invasive procedure has been deemed safe and effective for the management of ETD, care must be taken to ensure that patients are appropriately counseled on the possible risks. It is also crucial to counsel patients on strict sinus precautions and avoidance of activities which may increase endonasal pressure for at least 3 days postoperatively.

\section{Statement of Ethics}

IRB exemption was obtained for this case report, Protocol \# 2005022111. Per the Weill Cornell Medicine IRB on May 20, 2020: "The Office of Research Integrity has conducted a review of the abovementioned submission and determined that the activities described in this protocol do not constitute human subjects research as this is a case study of a single patient. As a result, 45 CFR part 46 does not apply. Therefore, neither IRB approval nor a notice of exemption is required for you to proceed with your project." Written informed consent was obtained from the patient for publication of this case report and any accompanying images.

\section{Conflict of Interest Statement}

Dr. Anaïs Rameau is a cofounder and Chief Executive Officer of MyophonX, a wearable device used to restore speech in patients with limited phonation capacity.

\section{Funding Sources}

The authors did not receive any funding.

\section{Author Contributions}

Sallie Long, cowrote, and edited the manuscript; Ade Obayemi, cowrote, and edited the manuscript; Anaïs Rameau, originated idea, cowrote, and edited the manuscript.

\section{References}

1 Huisman JML, Verdam FJ, Stegeman I, de Ru JA. Treatment of eustachian tube dysfunction with balloon dilation: a systematic review. Laryngoscope. 2018;128(1):237-47.

2 Adil E, Poe D. What is the full range of medi$\mathrm{cal}$ and surgical treatments available for patients with eustachian tube dysfunction? Curr Opin Otolaryngol Head Neck Surg. 2014; 22(1):8-15.
3 Skevas T, Dalchow CV, Euteneuer S, Sudhoff $\mathrm{H}$, Lehnerdt G. Cervicofacial and mediastinal emphysema after balloon eustachian tuboplasty (BET): a retrospective multicenter analysis. Eur Arch Otorhinolaryngol. 2018; 275(1):81-7.
$4 \mathrm{CPT}^{\star}$. Editorial summary of panel actions. Sep 2019.

5 Shah RR, Thomas WW, Naples JG, Ruckenstein MJ. Subcutaneous emphysema and pneumomediastinum after eustachian tube balloon dilation. Otolaryngol Head Neck Surg. 2018;159(1):203-5. 